\title{
A Novel Index Structure to Efficiently Match Events in Large-Scale Publish/Subscribe Systems
}

\author{
Jingli Yang*, Jing Fan, Chengyu Li, Shouda Jiang \\ Department of Automatic Testing and Control \\ Harbin Institute of Technology, Harbin 150080, China
}

\begin{abstract}
The event matching algorithm, which checks the events against all the subscriptions, is a fundamental component of large-scale content-based publish/subscribe systems, and it is the key issue for improving the efficiency of the entire system. To meet the increasing efficiency requirements of real-time publish/subscribe systems, an event matching algorithm named PADEM (Pairwise Attribute Division based Event Matching) is presented in this paper. By dividing the attribute space into multiple pairwise attribute subspaces, PADEM constructs a novel index structure to classify all subscriptions in systems. This index structure can guarantee the matching process in its each unit can only be triggered by corresponding events. The experimental results demonstrate that PADEM can dramatically improve the efficiency of event matching, particularly in large-scale distributed systems with high volumes of subscriptions.

Keywords: Content-based publish/subscribe, event matching, pairwise attribute division, matching time
\end{abstract}

\section{Introduction}

The development of Internet technology has changed the application and scale of distributed systems, which also brings higher requirements for the flexibility and scalability of such systems. The content-based publish/subscribe system has been widely applied in many applications, such as banking, e-commerce and e-government, because of the merits of asynchronous transmission, multi-point communications and loose couplings [1-4].

In a typical content-based publish/subscribe system, subscribers can declare some constraints on the content of the attributes [5]. Event matching is a process that brokers during the system check the event issued by publishers against each constraint of attributes to find the matched subscriptions. When there is a large volume of subscriptions and publishing events, brokers receive pressure from the event matching operation, which may reduce the efficiency of the system [6]. Therefore, the efficiency of the event matching algorithm is the key issue for large-scale content-based publish/subscribe systems.

\footnotetext{
${ }^{*}$ Corresponding author

Email address: jinglidg@hit.edu.cn (Jingli Yang)
} 
Currently, two types of strategies have been proposed for enhancing the matching efficiency of content-based publish/subscribe systems. The first type is to reduce the volume of subscriptions by using covering, merging, subsumption and partitioning. Subscription covering methods [7-11] are utilized to prevent useless dissemination of subscriptions. Merging methods [12,13] and subsumption methods [14-19] are proposed to reduce the volume of subscriptions. BE-Tree [20] partitions subscriptions using a space partitioning stage and a space clustering stage, and it can classify the subscriptions based on the ranges of the attributes. Opindex [21] is a two-level index structure that can support some subscription operators that are more complicated than BE-tree. PubSub [22] is another example of a partitioning-based system, and its architecture supports a large amount of operations and data types. However, a common disadvantage of these methods is the poor performance in cases where subscriptions are frequently updated, and the efforts expended to eliminate false positives can induce additional burdens.

The other type of event matching strategy is to use highly efficient index structures. SIENA [23, 24] and TAMA [25] are two typical event matching algorithms in this field. SIENA [23] uses a two-part forwarding table as the index structure, and it is not applicable for dynamic networks whose subscriptions are updated frequently because a minor modification of subscriptions always causes the reconstruction of the entire structure. TAMA [25] constructs a threelayer matching table to index the approximately matched subscriptions. One problem in both SIENA and TAMA is that although one constraint of the subscription is identified as unmatched, they still have to judge the remaining matched constraints. Hence, the performance of these algorithms decreases because a subscription always contains more than one constraint. H-Tree [26] constructs a hash table by mapping homogeneous subscriptions into the same bucket, which is built with the middle value of the range in constraints. Therefore, the matching efficiency is improved by filtering out the unmatched subscriptions in the matching process. Indeed, H-Tree performs well in cases of a large number of partially matched subscriptions, but its memory consumption is very high when there are a large amount of attributes in the attribute space. REIN [27] utilizes an index structure of multiple bucket lists, which are created by dividing ranges of attributes into several buckets, and it also retains the relationship between the buckets and the bucket lists. In REIN, the matched subscriptions are selected by marking the unmatched subscriptions first, which can avoid wasting time in counting matched constraints. However, the superiority of REIN is not obvious when the matched subscriptions are only a small fraction of the entire subscription set. Moreover, it is difficult to choose the best value for the amount of buckets since the performance of REIN decreases once the amount of buckets reaches a critical point. DMM [28] constructs a searching tree by mapping events and subscriptions to a multi-dimensional space, and it can shorten the time for finding the matched subscriptions stored in the corresponding nodes. However, DMM has to partition the multi-dimensional space by half many times, which leads to a long time consumption for constructing the tree structure. HEMA [29] detaches a few subscriptions from the mixed list to form a partial sequence table. It can meet the demands of systems in which the subscriptions are frequently updated, but the event matching efficiency does not significantly improve in the static case compared with its counterparts.

Aiming to overcome the shortcomings of existing algorithms, this paper proposes an event matching algorithm for improving the efficiency of content-based publish/subscribe systems. The innovative contributions of this paper are 
summarized as follows.

(1) A novel index structure is innovatively developed to divide the entire attribute space into multiple pairwise attribute subspaces, and each subspace consists of two buckets specified on its own attribute. All the subscriptions are classified to be stored in the buckets of the subspaces. This index structure can guarantee that the matching process in each unit (bucket of the pairwise attribute subspace) can only be triggered by corresponding events.

(2) Based on the newly developed index structure, an efficient event matching algorithm named PADEM (Pairwise Attribute Division based Event Matching) is proposed to resolve the performance degradation problem as the system scale increases.

(3) The performance metrics (e.g. matching time, insertion time, deletion time and memory consumption) of PADEM are theoretically analysed and compared with its counterparts.

(4) A series of experiments are designed to verify the performance of the proposed PADEM algorithm. The results of these experiments show that the performance of the proposed PADEM algorithm is better than that of other existing algorithms in this field.

The remainder of this paper is organized as follows. Section 2 introduces some definitions and research background. Section 3 presents the details of the proposed PADEM algorithm. Section 4 conducts the theoretical analysis of PADEM. To verify the performance of PADEM, experiments for various situations are conducted in Section 5. Finally, the conclusions are presented in Section 6.

\section{Definitions and Background}

\subsection{Definitions}

\subsubsection{Attribute Space}

An attribute space $\mathbf{A}=\left\{A_{1}, A_{2}, \ldots, A_{N}\right\}$ is an ordered $N$-tuple of attributes, where $N$ is the size of the attribute space. $A_{i}(1 \leq i \leq N)$ is the $i$-th attribute in $\mathbf{A}$, and it is sorted by certain rules. Any attribute that appears in an event or a subscription belongs to the attribute space.

\subsubsection{Events}

An event, which is also referred to as a message in some references, is an observable occurrence [27]. Classically, an event is defined as a combination of attribute-value pairs $\mathbf{E}=\left\{E_{r_{1}}, E_{r_{2}}, \ldots, E_{r_{M}}\right\}$, where $M$ is the number of attributes that appear in $\mathbf{E}, E_{r_{m}}=\left\{A_{r_{m}}, V_{r_{m}}\right\}\left(1 \leq m \leq M\right.$ and $\left.1 \leq r_{m} \leq N\right)$ is the $m$-th attribute-value pair in $\mathbf{E}$, and $A_{r_{m}}$ is the $r_{m}$-th attribute in the attribute space. In general, each attribute appears only once in an event expression. For example, $E=\{\{$ num, 100$\},\{$ weight, 63$\},\{$ height, 12$\}\}$ presents an event that contains three attributes whose names are num, weight and height, and their corresponding values are 100, 63 and 12, respectively. Without loss of generality, it is assumed that the attribute-value pairs in an event are sorted according to the order of the attributes in the attribute space; thus, $r_{1}<r_{2}<\ldots<r_{M}$. 


\subsubsection{Constraints}

A constraint is a qualification specified on an attribute selected from the attribute space [27]. In general, a constraint is denoted as $S_{i}=\left\{A_{i}, U B_{i}, L B_{i}\right\}$, where $U B_{i}$ and $L B_{i}$ are the upper bound and lower bound of the constraint specified on attribute $A_{i}$, respectively, and $\left[L B_{i}, U B_{i}\right]$ is also called the range of the constraint. For example, $S=\{$ weight $, 70,60\}$ specifies the attribute weight with a range $[60,70]$.

\subsubsection{Subscriptions}

A subscription is expressed as a combination of several constraints $\mathbf{S}=\left\{I D, S_{r_{1}}, S_{r_{2}}, \ldots S_{r_{C}}\right\}$, where $I D$ is the unique identification of the subscription and $C$ is the number of constraints contained in the subscription [27]. $S_{r_{c}}=$ $\left\{A_{r_{c}}, U B_{r_{c}}, L B_{r_{c}}\right\}\left(1 \leq c \leq C\right.$ and $\left.1 \leq r_{c} \leq N\right)$ is the $c$-th constraint specified on attribute $A_{r_{c}}$, and $A_{r_{c}}$ is the $r_{c}$-th attribute in the attribute space. The set of all subscriptions in a system can be expressed as $\mathbf{S u b}=\left\{\mathbf{S}^{1}, \mathbf{S}^{2}, \ldots, \mathbf{S}^{P}\right\}$, and $P$ is the number of subscriptions in the set. Without loss of generality, it is assumed that the constraints in a subscription are also sorted according to the order of the attributes in the attribute space; thus, $r_{1}<r_{2}<\ldots<r_{C}$.

\subsubsection{Event Matching}

A subscription matches an event if all the constraints contained in it are satisfied with the corresponding values of the attributes in the event [27]. Mathematically, a subscription $\mathbf{S}=\left\{I D, S_{r_{1}}, S_{r_{2}}, \ldots S_{r_{C}}\right\}\left(S_{r_{c}}=\left\{A_{r_{c}}, U B_{r_{c}}, L B_{r_{c}}\right\}\right.$, $1 \leq c \leq C)$ matches the event $\mathbf{E}=\left\{E_{r_{1}}, E_{r_{2}}, \ldots, E_{r_{M}}\right\}\left(E_{r_{m}}=\left\{A_{r_{m}}, V_{r_{m}}\right\}, 1 \leq m \leq M\right)$ when the following conditions hold:

$$
L B_{r_{j}} \leq V_{r_{j}} \leq U B_{r_{j}} \text {, s.t. } \forall j, 1 \leq j \leq C
$$

For a content-based publish/subscribe system that contains a set of subscriptions $\mathbf{S u b}=\left\{\mathbf{S}^{1}, \mathbf{S}^{2}, \ldots, \mathbf{S}^{P}\right\}$, the event matching process is to find all subscriptions from $\operatorname{Sub}$ that match $\mathbf{E}$. Denote $\operatorname{Sub}(\mathbf{E})$ as the set of matched subscriptions that is also a subset of Sub.

$$
\operatorname{Sub}(\mathbf{E})=\left\{\mathbf{S}^{p} \mid \mathbf{S}^{p} \in \mathbf{S u b} \cap \mathbf{S}^{p} \text { matches } \mathbf{E}\right\}
$$

\subsubsection{Pairwise Attribute Subspaces}

A pairwise attribute subspace $A_{i} A_{j}$ is a subset of the entire attribute space $\mathbf{A}$ that has two attributes $A_{i}$ and $A_{j}$ as neighbours, and the index of the second attribute is always larger than that of the first attribute $(i<j)$. Moreover, there are two buckets $A_{i} A_{j}\left[A_{i}\right]$ and $A_{i} A_{j}\left[A_{j}\right]$ in a pairwise attribute subspace, and each bucket corresponds to an attribute $\left(A_{i}\right.$ or $\left.A_{j}\right)$ of it.

\subsection{Publish/Subscribe Systems}

A typical publish/subscribe system is always composed of publishers (producers of information), subscribers (consumers of information) and the brokers. A publisher sends information (event) to the broker, a subscriber orders 
the information that it is interested in from the broker, and the broker matches events based on subscription information and routes the matched events to the subscribers. The brokers are responsible for dispatching the received events to the subscribers in a timely manner.

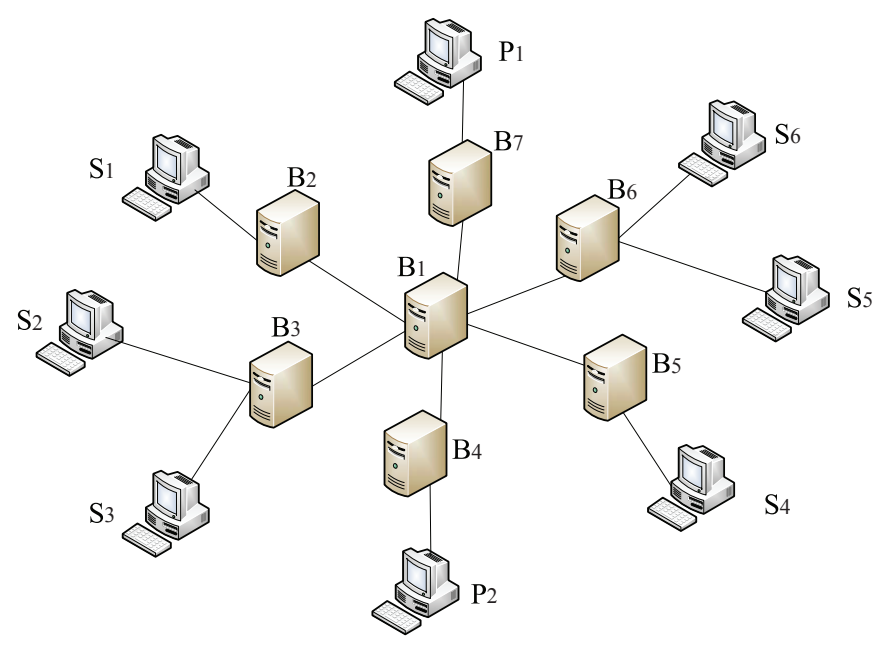

Figure 1: A publish/subscribe system composed of publishers/subscribers and brokers.

Figure 1 shows a typical publish/subscribe system, which consists of 6 subscribers, 2 publishers and 7 brokers. Event matching is performed on each broker to identify all subscribers that will receive the events. For example, when broker $\mathrm{B}_{3}$ receives an event from broker $\mathrm{B}_{1}$, an event matching process is conducted on broker $\mathrm{B}_{3}$ to deliver the event to the matched subscribers, $\mathrm{S}_{2}$ or $\mathrm{S}_{3}$.

\section{The Design of PADEM}

In this section, we describe the details about the design of PADEM. Specifically, a novel index structure based on pairwise attribute subspaces is proposed to address the invalid event matching process of content-based publish/subscribe systems. Furthermore, an example of the content-based publish/subscribe system is shown to demonstrate the index structure and the event matching process of PADEM.

\subsection{Overview}

The basic concept of PADEM is to design an index structure that can reduce the number of subscriptions involved in an event matching process to the greatest extent possible. It is known that the subscription is matched only in the case where all constraints in a subscription are satisfied by an event. Otherwise, the subscription is unmatched with the event. In the matching process, an event can only match subscriptions that contain the same attributes as itself, which means that subscriptions contain other attributes should not be involved in the event matching process. In theory, given an event $\mathbf{E}=\left\{E_{r_{1}}, E_{r_{2}}, \ldots, E_{r_{M}}\right\}\left(E_{r_{m}}=\left\{A_{r_{m}}, V_{r_{m}}\right\}, 1 \leq m \leq M, 1 \leq r_{m} \leq N\right)$ and a subscription 
$\mathbf{S}=\left\{I D, S_{r_{1}}, S_{r_{2}}, \ldots S_{r_{C}}\right\}\left(S_{r_{c}}=\left\{A_{r_{c}}, U B_{r_{c}}, L B_{r_{c}}\right\}, 1 \leq c \leq C, 1 \leq r_{c} \leq N\right)$, it is impossible for the event $\mathbf{E}$ to match the subscription $\mathbf{S}$ when there exists at least an attribute $A_{r_{j}}$ that is contained in $\mathbf{S}$ but does not appear in $\mathbf{E}$.

$$
A_{r_{j}} \in \mathbf{S} \cap A_{r_{j}} \notin \mathbf{E} \Rightarrow \mathbf{S} \notin \mathbf{S u b}(\mathbf{E}), \quad \text { s.t. } \exists j, 1 \leq j \leq C
$$

To utilize the filtering criteria of Eq. (3), a multi-layer index structure based on pairwise attribute subspaces is proposed. All subscriptions are classified and stored in units (buckets of the pairwise attribute subspaces) of the index structure. When an event is received by the broker, the event matching process is performed only on a few pairwise attribute subspaces. Thus, our objective of reducing the number of subscriptions involved in an event matching process can be achieved.

Suppose that the publish/subscribe system has an attribute space of four attributes $\mathbf{A}=\left\{A_{1}, A_{2}, A_{3}, A_{4}\right\}$; there are six pairwise attribute subspaces: $A_{1} A_{2}, A_{1} A_{3}, A_{1} A_{4}, A_{2} A_{3}, A_{2} A_{4}$ and $A_{3} A_{4}$. For an event $\mathbf{E}=\left\{E_{1}, E_{2}, E_{4}\right\}$ that has attributes $A_{1}, A_{2}$ and $A_{4}$, the matching process of event $\mathbf{E}$ is only performed on subspaces $A_{1} A_{2}, A_{2} A_{4}$ and $A_{1} A_{4}$, which means that many subscriptions can be excluded to improve the event matching efficiency.

\subsection{Division of Pairwise Attribute Subspaces}

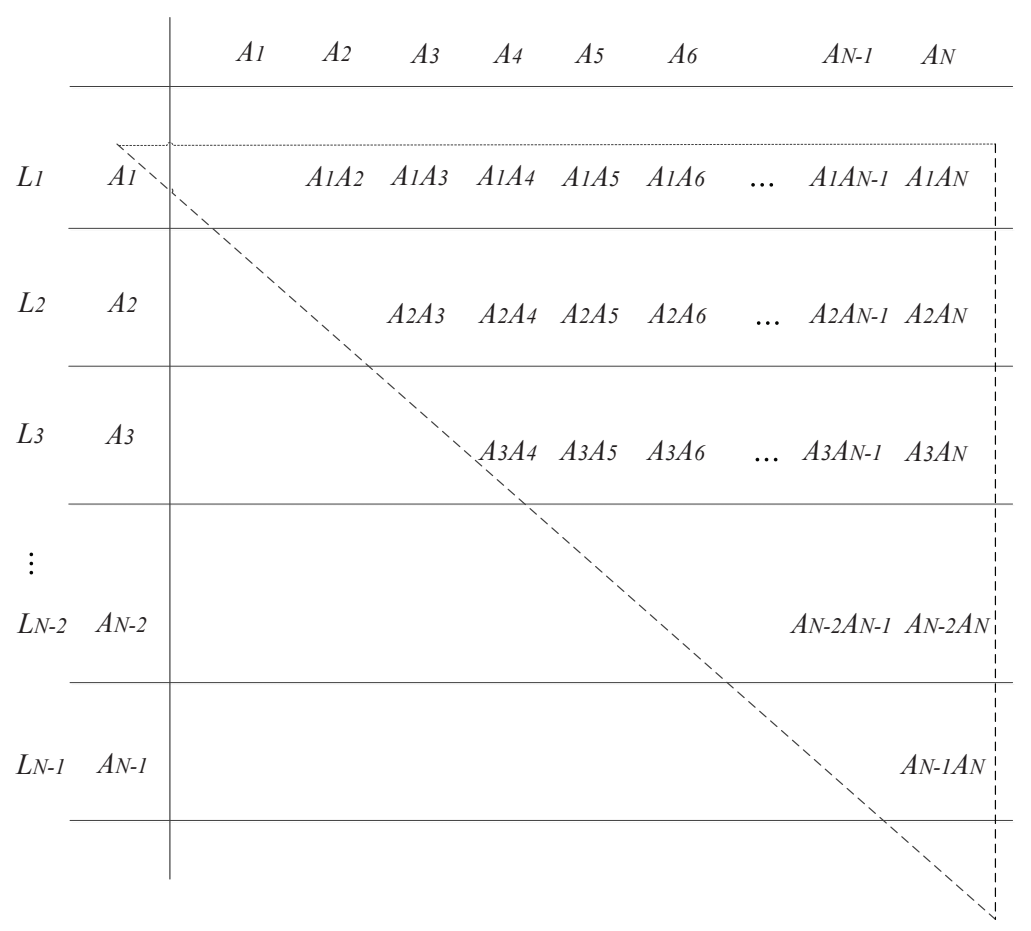

Figure 2: A multi-layer index structure composed of pairwise attribute subspaces.

Since the division of pairwise attribute subspaces is the key issue for the efficiency of our algorithm, more details about it are described in the following. Suppose that $\mathbf{A}=\left\{A_{1}, A_{2}, \ldots, A_{N}\right\}$ is the entire attribute space, and the subscript 
of each attribute represents its position in the attribute space. As shown in Figure 2, a multi-layer index structure that has $N-1$ layers is constructed for $\mathbf{A}$. In detail, for layer $L_{1}$, the first attribute $A_{1}$ is combined with the attribute ranked after it to construct multiple pairwise attribute subspaces, such as $A_{1} A_{2}, A_{1} A_{3}, A_{1} A_{4}$ and so on. In layer $L_{i}$, attribute $A_{i}$ is combined with attribute $A_{j}$ to construct the pairwise attribute subspace $A_{i} A_{j}$, where $A_{j}$ represents any attribute ranked after $A_{i}$. Hence, the number of pairwise attribute subspaces in layer $L_{i}$ is $N-i$, and the total number of subspaces in the structure is $N(N-1) / 2$. Finally, a multi-layer index structure can be constructed, and it is an upper triangle as shown in Figure 2.

For a pairwise attribute subspace $A_{i} A_{j}$, two buckets named $A_{i} A_{j}\left[A_{i}\right]$ and $A_{i} A_{j}\left[A_{j}\right]$ are constructed for attributes $A_{i}$ and $A_{j}$, respectively. The bucket $A_{i} A_{j}\left[A_{i}\right]$ stores the bounds of the constraints specified on $A_{i}$ and the $I D$ of subscriptions that contain $A_{i}$ and $A_{j}$ as neighbours. The bucket $A_{i} A_{j}\left[A_{j}\right]$ stores the bounds of constraints specified on $A_{j}$ and the $I D$ of subscriptions that not only contain $A_{i}$ and $A_{j}$ as neighbours but also have $A_{j}$ as its last attribute. To accelerate the event matching process, the upper and lower bounds of constraints are stored in ascending order, respectively. The details of a pairwise attribute subspace are shown in Figure 3. By using this rule, all subscriptions can be classified and stored in the corresponding buckets. Upon matching an event, the matched subscriptions can be quickly selected using the multi-layer index structure.

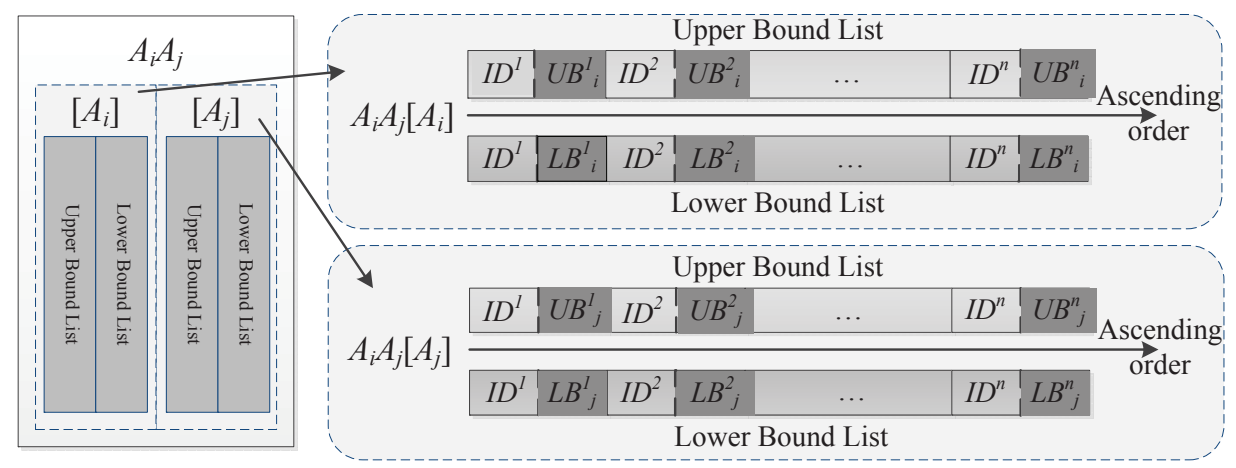

Figure 3: A pairwise attribute subspace $A_{i} A_{j}$.

\subsection{Arrangement of Subscriptions in PADEM}

Once the multi-layer index structure is constructed, the next task is to classify all subscriptions and store their information in units (buckets of the pairwise attribute subspaces) of the index structure.

Suppose that $\mathbf{S}=\left\{I D, S_{r_{1}}, S_{r_{2}}, \ldots, S_{r_{C}}\right\}$ is a subscription of the system, where $S_{r c}=\left\{A_{r_{c}}, U B_{r_{c}}, L B_{r_{c}}\right\}(1 \leq c \leq C$, $\left.1 \leq r_{c} \leq N\right)$ is the $c$-th constraint specified on attribute $A_{r_{c}}$ and $C$ is the number of constraints in it. In the constructed multi-layer index structure, the subscription $\mathbf{S}$ is stored in $C-1$ different pairwise attribute subspaces $A_{r_{c}} A_{r_{c+1}}(1 \leq$ $c \leq C-1$ ) for $C$ times. Therein, the pairwise attribute subspace $A_{r_{C-1}} A_{r_{C}}$ stores the information of the subscription $\mathbf{S}$ in its two buckets, while all other subspaces $A_{r_{c}} A_{r_{c+1}}(1 \leq c \leq C-2)$ store the information only in a bucket. Denote 
$U(\mathbf{S})$ as the set of buckets that store the information of $\mathbf{S}$, and it can be expressed as follows:

$$
U(\mathbf{S})=A_{r_{C-1}} A_{r_{C}}\left[A_{r_{C}}\right]+\bigcup_{c=1}^{C-1} A_{r_{c}} A_{r_{c+1}}\left[A_{r_{c}}\right]
$$

To reduce memory consumption, only the information of the constraint specified on the corresponding attribute and the $I D$ of the subscription are stored in each bucket of $U(\mathbf{S})$. In detail, the bucket $A_{r_{c-1}} A_{r_{c}}\left[A_{r_{c}}\right]$ stores $S_{r_{c}}=$ $\left\{A_{r_{c}}, U B_{r_{c}}, L B_{r_{c}}\right\}$ and the $I D$ by inserting the upper bound $U B_{r_{c}}$ and the $I D$ into its upper bound list and inserting the lower bound $L B_{r_{c}}$ and the $I D$ into its lower bound list in ascending order, respectively.

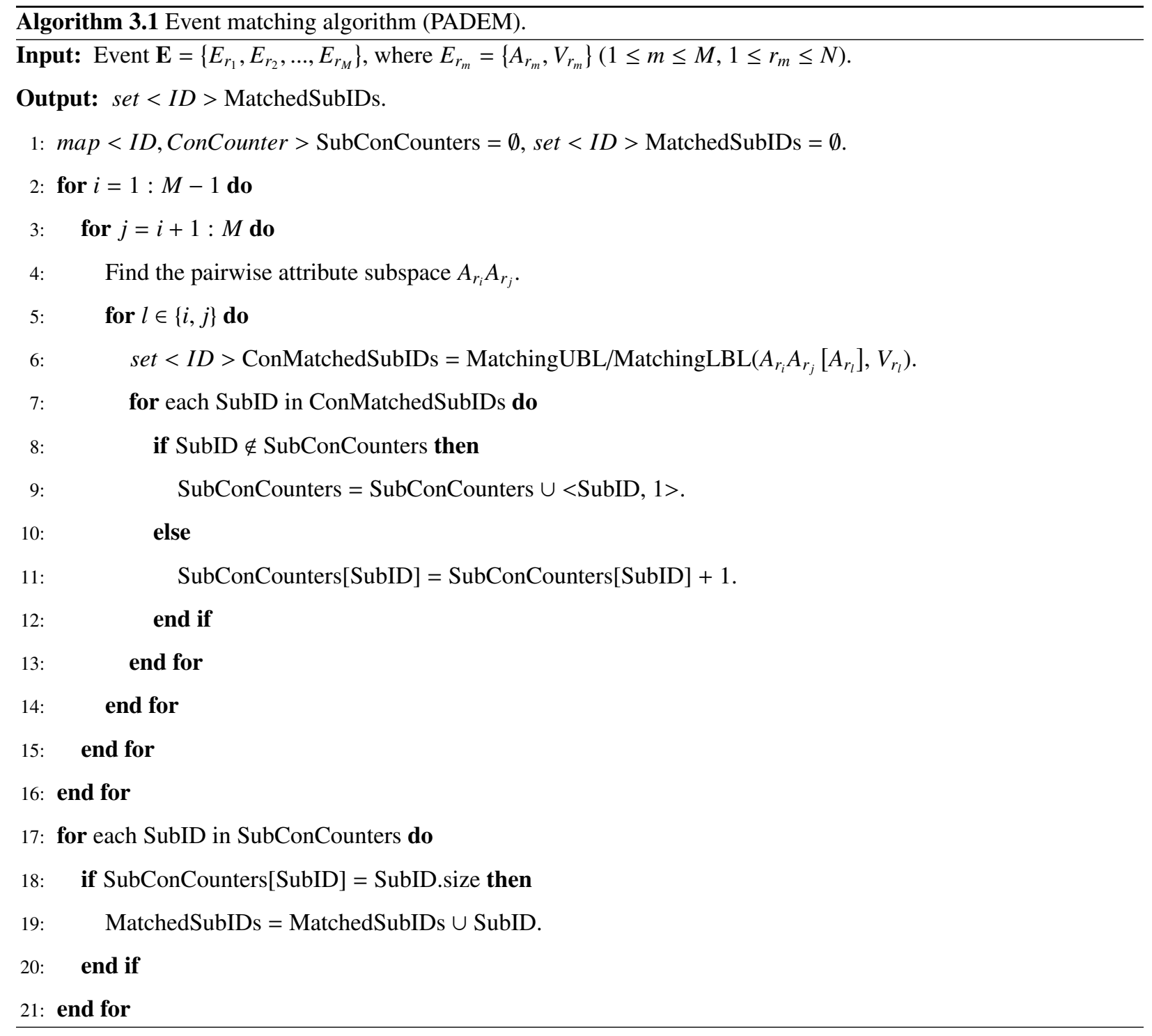




\subsection{Event Matching}

In an event matching process, each event is routed straightforward to the likely matched subscriptions through the multi-layer index structure constructed above. First, a map structure named map $<$ ID, ConCounter $>$ SubConCounters is initialized to count the matched constraints of subscriptions (line 1). Upon the arrival of an event $\mathbf{E}=\left\{E_{r_{1}}, E_{r_{2}}, \ldots, E_{r_{M}}\right\}\left(E_{r_{m}}=\left\{A_{r_{m}}, V_{r_{m}}\right\}, 1 \leq m \leq M, 1 \leq r_{m} \leq N\right)$, a number of $M(M-1) / 2$ pairwise attribute subspaces that store possibly matched subscriptions are found based on the neighbourhood of the attributes that are contained in event $\mathbf{E}$ (lines 2-3). For each of the above pairwise attribute subspaces $A_{r_{i}} A_{r_{j}}(1 \leq i<j \leq M$, $\left.1 \leq r_{i}<r_{j} \leq N\right)$ (line 4), the event matching process is performed on its two buckets $A_{r_{i}} A_{r_{j}}\left[A_{r_{i}}\right]$ and $A_{r_{i}} A_{r_{j}}\left[A_{r_{j}}\right]$. For each bucket $A_{r_{i}} A_{r_{j}}\left[A_{r_{l}}\right](l \in\{i, j\})$, the bisect searching algorithm is employed on its two lists (upper bound list and lower bound list), respectively. Therefore, the subscriptions that satisfy one of the following conditions are involved in the event matching process (line 6).

(C.1) the upper bound of the constraint specified on the attribute $A_{r_{l}}$ is larger than $V_{r_{l}}$ in $\mathbf{E}$.

(C.2) the lower bound of the constraint specified on the attribute $A_{r_{l}}$ is less than $V_{r_{l}}$ in $\mathbf{E}$.

Regarding the counting-based algorithm, the ConCounter of those subscriptions is increased by 1 (line 11). After all of the above subspaces are processed, the subscriptions whose ConCounter is equal to the SubID.size (SubID.size is twice the number of constraints in this subscription) are matched with the event (lines 17-20). The event matching process of PADEM is shown in Algorithm 3.1.

\subsection{Example}

In this section, an example of the content-based publish/subscribe system is presented to demonstrate the index structure and the event matching process of PADEM. There are five attributes in attribute space A, and ten subscriptions are waiting for event matching, as described in Table 1. Here, the value of each attribute is assumed to range from 0 to 20 .

Table 1: List of ten subscriptions

\begin{tabular}{ccccccccccc}
\hline Subscriptions & $\mathbf{S}^{0}$ & $\mathbf{S}^{1}$ & $\mathbf{S}^{2}$ & $\mathbf{S}^{3}$ & $\mathbf{S}^{4}$ & $\mathbf{S}^{5}$ & $\mathbf{S}^{6}$ & $\mathbf{S}^{7}$ & $\mathbf{S}^{8}$ & $\mathbf{S}^{9}$ \\
\hline$I D$ & 0 & 1 & 2 & 3 & 4 & 5 & 6 & 7 & 8 & 9 \\
$A_{1}$ & {$[3,7]$} & {$[7,12]$} & {$[2,9]$} & - & {$[17,19]$} & - & - & {$[2,13]$} & {$[6,11]$} & - \\
$A_{2}$ & {$[2,8]$} & {$[2,7]$} & - & {$[7,13]$} & {$[3,9]$} & {$[16,18]$} & - & - & - & {$[8,11]$} \\
$A_{3}$ & - & {$[1,8]$} & {$[13,16]$} & {$[3,6]$} & - & {$[13,17]$} & {$[11,19]$} & - & {$[11,14]$} & - \\
$A_{4}$ & {$[9,14]$} & - & {$[6,10]$} & - & - & {$[4,9]$} & {$[4,16]$} & {$[7,12]$} & - & {$[2,4]$} \\
$A_{5}$ & - & - & - & {$[8,17]$} & {$[12,18]$} & - & {$[4,14]$} & {$[15,20]$} & {$[13,18]$} & {$[2,13]$} \\
\hline
\end{tabular}

By using the PADEM algorithm, a multi-layer index structure consisting of ten pairwise attribute subspaces is constructed as described in Section 3.2. When classifying a subscription, its information is stored in some units of 

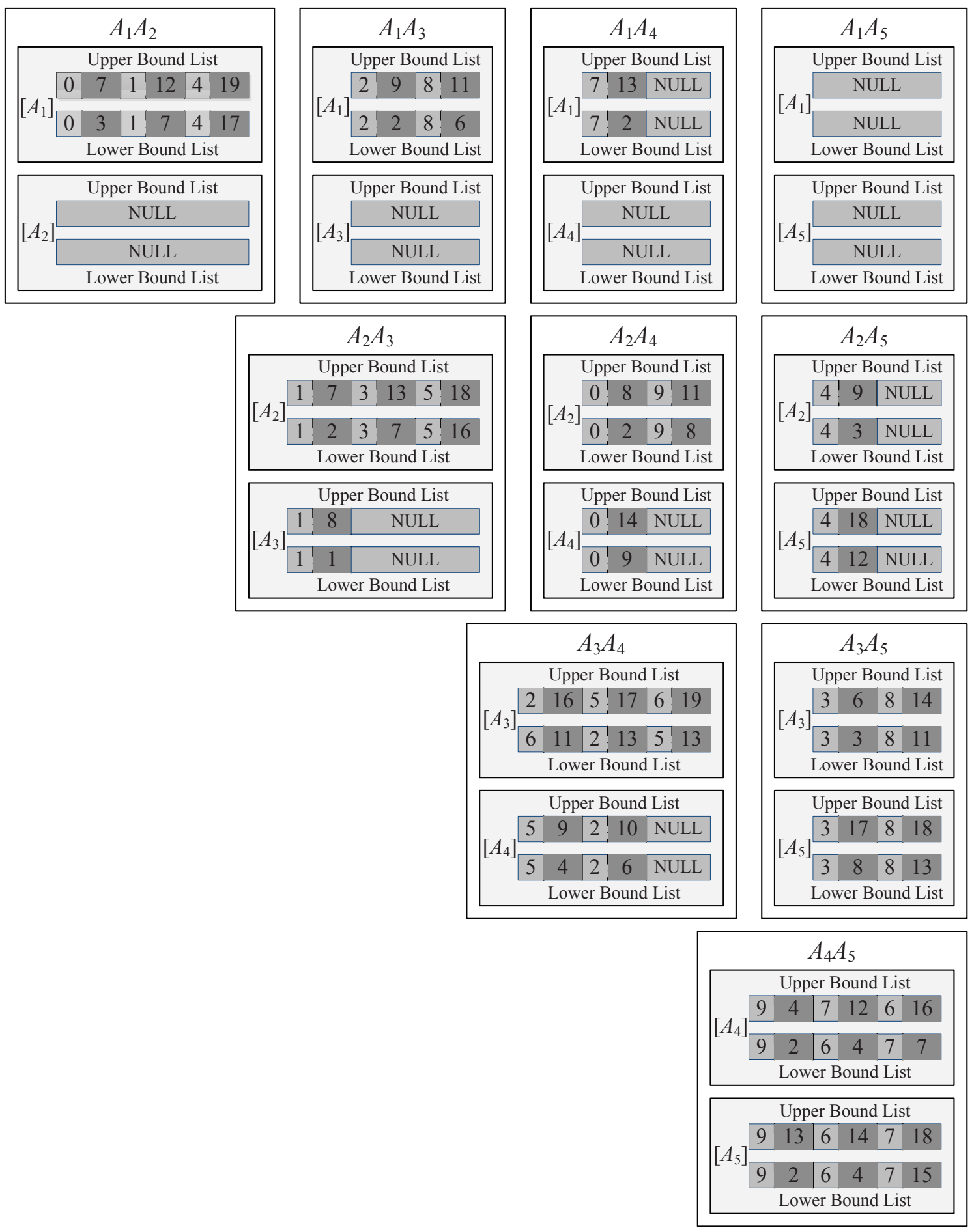

Figure 4: The arrangement of ten subscriptions in the multi-layer index structure based on pairwise attribute subspaces. 
the structure according to the rules defined in Section 3.3. For example, when processing the subscription $\mathbf{S}^{0}=$ $\left\{I D, S_{1}, S_{2}, S_{4}\right\}=\left\{0,\left\{A_{1}, 7,3\right\},\left\{A_{2}, 8,2\right\},\left\{A_{4}, 14,9\right\}\right\}$, its information is stored in a bucket $\left(A_{1} A_{2}\left[A_{1}\right]\right)$ of subspace $A_{1} A_{2}$ and two buckets $\left(A_{2} A_{4}\left[A_{2}\right]\right.$ and $\left.A_{2} A_{4}\left[A_{4}\right]\right)$ of subspace $A_{2} A_{4}$. In detail, the lower bound of constraint $S_{1}$ specified on attribute $A_{1}$ (which is 3 ) and the $I D$ of the subscription $\mathbf{S}^{0}$ (which is 0 ) are inserted into the lower bound list of bucket $A_{1} A_{2}\left[A_{1}\right]$. Similarly, the upper bound of constraint $S_{1}$ specified on attribute $A_{1}$ (which is 7 ) and the $I D$ of the subscription $\mathbf{S}^{0}$ (which is 0 ) are inserted into the upper bound list of bucket $A_{1} A_{2}$ [ $\left.A_{1}\right]$. Since $A_{4}$ is the last attribute contained in $\mathbf{S}^{0}$, the bound values of constraint $S_{2}$ specified on attribute $A_{2}$ (which are 8 and 2) and those of constraint $S_{4}$ specified on attribute $A_{4}$ (which are 14 and 9) are stored in buckets $A_{2} A_{4}\left[A_{2}\right]$ and $A_{2} A_{4}\left[A_{4}\right]$ of subspace $A_{2} A_{4}$, respectively. By parity of reasoning, ten subscriptions are stored in this system, as shown in Figure 4.

Upon the arrival of an event $\mathbf{E}=\left\{A_{1}=5, A_{2}=4, A_{4}=13, A_{5}=19\right\}$, PADEM determines all pairwise attribute subspaces that store possible matched subscriptions according to the neighbourhood of the attributes that appear in this event, and six pairwise attribute subspaces are achieved as follows: $A_{1} A_{2}, A_{1} A_{4}, A_{1} A_{5}, A_{2} A_{4}, A_{2} A_{5}$ and $A_{4} A_{5}$. Then, the event matching process of event $\mathbf{E}$ is performed in those subspaces.

Let us take the subspace $A_{1} A_{2}$ as an example. PADEM searches the lower bound list of bucket $A_{1} A_{2}\left[A_{1}\right]$ according to the corresponding value $A_{1}=6$ in the event $\mathbf{E}$, and $\mathbf{S}^{0}$ is involved in the event matching process because its lower bound of the constraint specified on attribute $A_{1}$ is less than 6 . Therefore, the ConCounter of the subscription $\mathbf{S}^{0}$ is increased by 1 . Similarly, PADEM continues to search the upper bound list in bucket $A_{1} A_{2}\left[A_{1}\right]$ according to the corresponding value $A_{1}=6$, and $\mathbf{S}^{0}, \mathbf{S}^{1}$, and $\mathbf{S}^{4}$ are involved in the event matching process because their upper bounds of the constraints specified on attribute $A_{1}$ are larger than 6. Therefore, the ConCounter of each of these subscriptions is increased by 1 . By using the same regulation, all subscriptions involved in the event matching process of PADEM are listed in Table 2:

Table 2: Subscriptions involved in the event matching process of PADEM.

\begin{tabular}{cccccccccccccccc}
\hline Subspaces & \multicolumn{2}{c}{$A_{1} A_{2}$} & \multicolumn{2}{c}{$A_{1} A_{4}$} & \multicolumn{2}{c}{$A_{1} A_{5}$} & \multicolumn{2}{c}{$A_{2} A_{4}$} & \multicolumn{2}{c}{$A_{2} A_{5}$} & \multicolumn{3}{c}{$A_{4} A_{5}$} \\
\hline Buckets & {$\left[A_{1}\right]$} & {$\left[A_{2}\right]$} & {$\left[A_{1}\right]$} & {$\left[A_{4}\right]$} & {$\left[A_{1}\right]$} & {$\left[A_{5}\right]$} & {$\left[A_{2}\right]$} & {$\left[A_{4}\right]$} & {$\left[A_{2}\right]$} & {$\left[A_{5}\right]$} & {$\left[A_{4}\right]$} & {$\left[A_{5}\right]$} \\
\hline Upper Bound List & $\mathbf{S}^{0}, \mathbf{S}^{1}, \mathbf{S}^{4}$ & - & $\mathbf{S}^{7}$ & - & - & - & $\mathbf{S}^{0}, \mathbf{S}^{9}$ & $\mathbf{S}^{0}$ & $\mathbf{S}^{4}$ & - & $\mathbf{S}^{6}$ & $\mathbf{S}^{7}$ \\
Lower Bound List & $\mathbf{S}^{0}$ & - & $\mathbf{S}^{7}$ & - & - & - & $\mathbf{S}^{0}$ & $\mathbf{S}^{0}$ & $\mathbf{S}^{4}$ & $\mathbf{S}^{4}$ & $\mathbf{S}^{6}, \mathbf{S}^{7}, \mathbf{S}^{9}$ & $\mathbf{S}^{6}, \mathbf{S}^{7}, \mathbf{S}^{9}$ \\
Sum of ConCounter & 4 & 0 & 2 & 0 & 0 & 0 & 3 & 2 & 2 & 1 & 4 & 4 \\
\hline
\end{tabular}

As shown in Table 2, only six subscriptions, namely, $\mathbf{S}^{0}, \mathbf{S}^{1}, \mathbf{S}^{4}, \mathbf{S}^{6}, \mathbf{S}^{7}$ and $\mathbf{S}^{9}$, are involved in the event matching process. Therefore, the proposed multi-layer index structure based on pairwise attribute subspaces can help to filter out subscriptions that are stored in irrelevant subspaces (such as $\mathbf{S}^{2}$ and $\mathbf{S}^{8}$ in subspace $A_{1} A_{3}$ and $\mathbf{S}^{3}$ and $\mathbf{S}^{5}$ in subspace $A_{2} A_{3}$ ). After the matching process is finished on the above six subspaces, $\mathbf{S}^{0}$ is found as being matched with event $\mathbf{E}$ because its ConCounter is equal to 6 (twice the number of the constraints in $\mathbf{S}^{0}$ ). 
Compared with PADEM, SIENA [23, 24] and REIN [27] classify all subscriptions via attributes; therefore, all subscriptions that have constraints specified on each attribute of the event may be involved the event matching process. As described in [23], SIENA finds matched subscriptions by counting the number of matched constraints, whereas REIN [27] determines all matched subscriptions by marking all unmatched constraints. When matching event E, all subscriptions involved in the event matching processes of SIENA and REIN are listed in Table 3 and Table 4.

\begin{tabular}{ccccc}
\multicolumn{5}{c}{ Table 3: Subscriptions involved in the event matching process of SIENA. } \\
\hline Attributes & $A_{1}$ & $A_{2}$ & $A_{4}$ & $A_{5}$ \\
\hline Upper Bound List & $\mathbf{S}^{0}, \mathbf{S}^{1}, \mathbf{S}^{2}, \mathbf{S}^{4}, \mathbf{S}^{7}, \mathbf{S}^{8}$ & $\mathbf{S}^{0}, \mathbf{S}^{1}, \mathbf{S}^{3}, \mathbf{S}^{4}, \mathbf{S}^{5}, \mathbf{S}^{9}$ & $\mathbf{S}^{0}, \mathbf{S}^{6}$ & $\mathbf{S}^{7}$ \\
Lower Bound List & $\mathbf{S}^{0}, \mathbf{S}^{2}, \mathbf{S}^{7}$ & $\mathbf{S}^{0}, \mathbf{S}^{1}, \mathbf{S}^{4}$ & $\mathbf{S}^{0}, \mathbf{S}^{2}, \mathbf{S}^{5}, \mathbf{S}^{6}, \mathbf{S}^{7}, \mathbf{S}^{9}$ & $\mathbf{S}^{3}, \mathbf{S}^{4}, \mathbf{S}^{6}, \mathbf{S}^{7}, \mathbf{S}^{8}, \mathbf{S}^{9}$ \\
Sum of ConCounter & 9 & 9 & 8 & 7 \\
\hline
\end{tabular}

Table 4: Subscriptions involved in the event matching process of REIN.

\begin{tabular}{cccccc}
\hline Attributes & $A_{1}$ & $A_{2}$ & $A_{3}$ & $A_{4}$ & $A_{5}$ \\
\hline Upper Bound Bucket List & - & - & $\mathbf{S}^{1}, \mathbf{S}^{2}, \mathbf{S}^{3}, \mathbf{S}^{5}, \mathbf{S}^{6}, \mathbf{S}^{8}$ & $\mathbf{S}^{2}, \mathbf{S}^{5}, \mathbf{S}^{7}, \mathbf{S}^{9}$ & $\mathbf{S}^{3}, \mathbf{S}^{4}, \mathbf{S}^{6}, \mathbf{S}^{8}, \mathbf{S}^{9}$ \\
Lower Bound Bucket List & $\mathbf{S}^{1}, \mathbf{S}^{4}, \mathbf{S}^{8}$ & $\mathbf{S}^{3}, \mathbf{S}^{5}, \mathbf{S}^{9}$ & $\mathbf{S}^{1}, \mathbf{S}^{2}, \mathbf{S}^{3}, \mathbf{S}^{5}, \mathbf{S}^{6}, \mathbf{S}^{8}$ & - & - \\
Sum of ConCounter & 3 & 3 & 12 & 4 & 5 \\
\hline
\end{tabular}

As shown in Table 3 and Table 4, the numbers of subscriptions involved in the event matching processes of SIENA and REIN are ten and nine, respectively, which are more than that of PADEM. Therefore, our proposed PADEM algorithm can reduce the number of subscriptions involved in the event matching process. Note that the efficiency of REIN decreases when the number of attributes in the attribute space is larger than the number of attributes in events. This decreased efficiency is because REIN has to mark all subscriptions that have constraints specified on the attributes that do not appear in the event. For example, REIN has to mark subscriptions $\left(\mathbf{S}^{1}, \mathbf{S}^{2}, \mathbf{S}^{3}, \mathbf{S}^{5}, \mathbf{S}^{6}\right.$, and $\left.\mathbf{S}^{8}\right)$ because these subscriptions have constraints specified on attribute $A_{3}$, but $A_{3}$ does not appear in event $\mathbf{E}$.

In addition, counting the number of constraints that are satisfied with the event is the most time-consuming operation in an event matching process, and it can be expressed using the sum of ConCounter in Algorithm 3.1. As shown in the last rows of Table 2, Table 3 and Table 4, the sum of ConCounter of PADEM (i.e. 22) for matching event $\mathbf{E}$ is less than that of SIENA (i.e. 33) and REIN (i.e. 27). Therefore, PADEM is more efficient in terms of event matching than SIENA and REIN.

\section{Theoretical Performance Analysis}

In this section, our proposed PADEM algorithm is analysed in terms of matching time, insertion time, deletion time and memory consumption. Table 5 summarizes the key parameters used in the following discussion. Because 
SIENA [23, 24] and REIN [27] share a common broker overlay with PADEM, they are also theoretically analysed to determine the applicability of the PADEM algorithm.

In addition to all the parameters listed in Table 5, the distribution of attributes in subscriptions and the distribution of constraint values can also affect the performance of the event matching process. Considering that the uniform distribution is the most universal model for generating the constraint values and the attributes in subscriptions $[8,10$, $20,22,26-28]$, our theoretical analysis is conducted with the following fundamental statistical assumptions about the subscriptions.

(S.1) The attributes in subscriptions are selected uniformly from the attribute space.

(S.2) The constraint values are selected uniformly from the value range.

Based on the above assumptions, we can simplify the theoretical analysis process and achieve explicit results upon comparison. However, some other types of distributions are also adopted in our following experiments to verify the adaptability of the proposed PADEM algorithm. For example, the Zipf model [26] is adopted in conjunction with the uniform model to evaluate the effects of the distribution of attributes in subscriptions. Similarly, the normal model and the Pareto model $[26,27]$ are also used together with the uniform model to evaluate the effects of the distribution of constraint values.

Table 5: The parameters used in the theoretical analysis and experiments.

\begin{tabular}{ccc}
\hline Name & Meaning & Value \\
\hline$N$ & the number of attributes in the attribute space & $50-100$ \\
$P$ & the number of subscriptions & $200 \mathrm{k}-2000 \mathrm{k}$ \\
$M$ & the number of attributes in events & $10-20$ \\
$C$ & the number of constraints in subscriptions & $5-15$ \\
$w$ & the width of range constraints & $0.1-0.9$ \\
$b$ & the number of buckets in REIN & 1500 \\
$d$ & the number of discretization levels in TAMA & 13 \\
\hline
\end{tabular}

\subsection{Matching Time}

As described in references [23-27], the most time-consuming operation in an event matching algorithm is counting the number of matched constraints (SIENA [23, 24], TAMA [25]) or marking the number of unmatched constraints (REIN [27]). Therefore, the number of subscriptions satisfying (C.1) or (C.2) in Section 3.4 is used as the metric for evaluating the matching time of SIENA and our proposed PADEM algorithms, whereas the number of subscriptions not satisfying (C.1) or (C.2) is used as the metric for evaluating the matching time of REIN. For each attribute $A_{i}$ $(1 \leq i \leq N)$ in the attribute space, suppose that $\mathbf{S}$ is a subscription that has a constraint specified on $A_{i}$ and $\mathbf{E}$ is 
an event that contains $A_{i}$ in one of its attribute-value pairs. According to (S.2), the probability of $\mathbf{S}$ satisfying (C.1) or $(\mathrm{C} .2)$ is $(1+w) / 2$, where $w$ is the width of range constraints that has been normalized on [0,1.0]. Similarly, the probability of $\mathbf{S}$ not satisfying (C.1) or (C.2) is $(1-w) / 2$.

SIENA is a typical index structure for event matching, and it consists of two parts [23]. The left-hand part is an index of constraints, and the right-hand part merges the combination of constraints into filters. In theory, SIENA contains $N$ individual buckets, and each of these buckets corresponds to an attribute in the attribute space. As described in [23], the constraints of each attribute are ordered according to their bound values to accelerate the event matching process, which means that an upper bound list and a lower bound list are contained in each bucket to store the upper bounds and lower bounds of the constraints in ascending order, respectively. According to (S.1), the number of subscriptions stored in each bucket is $P \times C / N$, where $P$ is the number of subscriptions in the system, $C$ is the number of constraints in subscriptions, and $N$ is the number of attributes in the attribute space. Based on the probability analysis in the previous paragraph, the number of subscriptions satisfying (C.1) or (C.2) in each bucket is $(P \times C / N) \times$ $((1+w) / 2) \times 2$. For an event that contains $M$ attributes, the event matching process is performed on the same number of buckets. Therefore, the complexity of the matching time of SIENA is

$$
T_{\text {SIENA }}=O((P \times C / N) \times(1+w) \times M)
$$

The index structure of REIN contains $2 N$ individual bucket lists, and each pair of them (a pair consists of a lower bound bucket list and an upper bound bucket list) corresponds to an attribute in the attribute space. In contrast to SIENA, each bucket list of REIN is divided into a series of buckets using the interval partition of the attribute values. This improvement can reduce the time required to find matched constraints from $O((\log (P \times C / N)) \times M)$ to $O((\log ((P \times C) /(b / 2))) \times M)$ because $b$ (the number of buckets in REIN) is considerably larger than $N$. However, REIN still exhibits a highly complex matching time because counting the number of matched constraints is more timeconsuming than finding matched constraints. According to (S.1), the number of subscriptions stored in each bucket list of REIN is $P \times C / N$. As described in [27], REIN marks all subscriptions not satisfying (C.1) or (C.2); thus, for each pair of bucket lists corresponding to an attribute that appears in the event, a number of $(P \times C / N) \times((1-w) / 2) \times 2$ subscriptions are marked as unmatched with the event. In addition, for each bucket list corresponding to an attribute that does not appear in the event, all subscriptions stored in it should be marked as unmatched with the event. For an event that contains a number of $M$ attributes, the event matching process of REIN is performed on its $2 N$ bucket lists. Therefore, the complexity of the matching time of REIN is

$$
T_{R E I N}=O((P \times C / N) \times(1-w) \times M+(P \times C / N) \times(N-M))
$$

Although the event matching process is conducted on $M(M-1)$ buckets of $M(M-1) / 2$ subspaces, the number of subscriptions satisfying (C.1) or (C.2) in PADEM is relatively few. This is because all subscriptions in PADEM are classified and stored in different units (buckets of the pairwise attribute subspaces) of the multi-layer index structure, which can guarantee that the event matching process is only performed on some relevant units. For each attribute $A_{i}$ 
in the attribute space, all subscriptions that have constraints specified on $A_{i}$ are classified and stored in $N-1$ buckets, which can be expressed as follows:

$$
\mathrm{H}\left(A_{i}\right)=\bigcup_{j=1}^{i-1} A_{j} A_{i}\left[A_{i}\right]+\bigcup_{k=i+1}^{N} A_{i} A_{k}\left[A_{i}\right]
$$

For an event $\mathbf{E}=\left\{E_{r_{1}}, E_{r_{2}}, \ldots, E_{r_{M}}\right\}\left(E_{r_{m}}=\left\{A_{r_{m}}, V_{r_{m}}\right\}, 1 \leq m \leq M, 1 \leq r_{m} \leq N\right)$ that contains $A_{i}$ in one of its attribute-value pairs, the event matching process on $A_{i}$ is only performed on a subset of $\mathrm{H}\left(A_{i}\right)$, which can be expressed as follows:

$$
\Gamma\left(A_{i}\right)=\bigcup_{\substack{m=1 \\ r_{m}<i}}^{M} A_{r_{m}} A_{i}\left[A_{i}\right]+\bigcup_{\substack{m=1 \\ r_{m}>i}}^{M} A_{i} A_{r_{m}}\left[A_{i}\right]
$$

Clearly, there are $M-1$ buckets in $\Gamma\left(A_{i}\right)$. According to (S.1) and (S.2), the number of subscriptions that have constraints specified on $A_{i}$ and satisfy (C.1) or (C.2) is $(P \times C / N) \times(1+w) \times((M-1) /(N-1))$. Therefore, the complexity of the matching time of PADEM is

$$
T_{P A D E M}=O((P \times C / N) \times(1+w) \times((M-1) /(N-1)) \times M)
$$

Considering that in a large-scale content-based publish/subscribe system, the number of attributes in the attribute space is always substantially larger than the number of attributes in the event $(N \gg M)$, the proposed PADEM algorithm has considerably more attractive performance in matching efficiency than SIENA and REIN.

\subsection{Insertion Time}

In SIENA, the information of a subscription is stored in $C$ buckets of the index structure, where $C$ is the number of attributes in subscriptions. Assume that $P$ subscriptions have been stored in the index structure; according to (S.1), the number of subscriptions stored in each bucket is $P \times C / N$, where $N$ is the number of attributes in the attribute space. When inserting a new subscription into a bucket of the index structure, the binary search method is employed on the upper bound list and the lower bound list to locate the position of the new subscription; thus, the time consumption in each bucket is $2 \times \log (P \times C / N)$. Therefore, the complexity of the insertion time of SIENA is $O(2 \times C \times \log (P \times C / N))$.

In REIN, the information of a subscription is stored in $2 C$ bucket lists of the index structure. In fact, each bucket list uses one of its buckets to store the information of the subscription. Assume that $P$ subscriptions are stored in the index structure with $b$ buckets. According to (S.1), the number of subscriptions stored in each bucket is $P \times C /(b / 2)$. When inserting a new subscription into a bucket of the index structure, the binary search method is employed to locate the position of the new subscription with a time consumption of $\log (P \times C /(b / 2))$. Therefore, the complexity of the insertion time of REIN is $O(2 \times C \times \log (P \times C /(b / 2)))$.

As described in Section 3.3, the information of a subscription is stored in $C$ buckets of the multi-layer index structure. Assume that $P$ subscriptions have been stored in the index structure; according to (S.1), the number of 
subscriptions stored in each bucket is $P \times C /(N \times(N-1))$. When inserting a new subscription into a bucket of the index structure, the binary search method is employed on the upper bound list and the lower bound list to locate the position of the new subscription; thus, the time consumption in each bucket is $2 \times \log (P \times C /(N \times(N-1)))$. Therefore, the complexity of the insertion time of PADEM is $O(2 \times C \times \log (P \times C /(N \times(N-1))))$.

In summary, our proposed PADEM algorithm achieves better performance than SIENA in terms of insertion time. Note that REIN can reduce the insertion time by increasing the number of buckets $b$. However, this also results in an increase in the matching time because the cost to traverse buckets increases with the number of buckets [27].

\subsection{Deletion Time}

The deletion of an existing subscription is similar to the insertion of a new subscription, which also affects $C$ buckets in SIENA and PADEM and $2 C$ bucket lists in REIN. The most time-consuming process of deletion is to go through each bucket list or bucket and find the position of the existing subscription using its $I D$. Therefore, the complexity of the deletion time of SIENA, REIN and PADEM is $O(2 \times C \times(P \times C / N)), O(2 \times C \times(P \times C /(b / 2)))$ and $O(2 \times C \times(P \times C /(N \times(N-1))))$, respectively.

\subsection{Memory Consumption}

Memory consumption in an event matching algorithm consists of three parts: structure information, subscription information and other information.

The structure information includes all units used for construction of the index structure. In SIENA, the index structure consists of $N$ buckets, and each bucket contains an upper bound list and a lower bound list. Suppose that each bucket and bound list occupy 4 bytes, respectively; then, the memory consumption of the structure information of SIENA is $12 N$. The index structure of REIN consists of $2 N$ bucket lists and $b$ buckets. Suppose that each bucket list and bucket occupy 4 bytes; then, the memory consumption of the structure information of REIN is $8 N+4 b$. For PADEM, the index structure is composed of $N(N-1) / 2$ subspaces, each subspace contains two buckets, and each bucket consists of an upper bound list and a lower bound list. If each subspace, bucket and bound list occupy 4 bytes, respectively, then the memory consumption of the structure information of PADEM is $14 N(N-1)$.

The subscription information is composed of bounds of constraints and the $I D$ of subscriptions, and each bound of the constraint and $I D$ of the subscription occupy 4 bytes. In SIENA, REIN and PADEM, the information of a subscription is stored $C$ times, and the upper bound or lower bound is used combined with the $I D$ of the subscription for each time of storage. Therefore, the memory consumption of the subscription information of all those algorithms is $16 P \times C$.

Note that SIENA and PADEM are both types of counting-based algorithms, which means that the number of constraints contained in each subscription is an essential condition for judging the matched subscriptions. When the number of constraints contained in each subscription occupies one byte, then these algorithms need $P$ bytes to store this other information. The details of memory consumption are listed in Table 6. 
Table 6: Details of memory consumption

\begin{tabular}{cccc}
\hline Item & SIENA & REIN & PADEM \\
\hline Structure information & $12 N$ & $8 N+4 b$ & $14 N(N-1)$ \\
Subscription information & $16 P \times C$ & $16 P \times C$ & $16 P \times C$ \\
Other information & $P$ & - & $P$ \\
\hline
\end{tabular}

\section{Experimental Results and Analysis}

To verify the efficiency of the Pairwise Attribute Division based Event Matching algorithm (PADEM), a series of experiments were conducted. Four metrics, namely, matching time, insertion time, deletion time, and memory consumption, were chosen to comprehensively verify the performance of PADEM.

\subsection{Experimental Setup}

All experiments were conducted on a computer with a Core i7-2640 CPU and 4 GB memory. The results of the performance evaluation are presented in the following parts. Three representative algorithms, namely, SIENA [23, 24], TAMA [25], and REIN [27], were used to compare with the proposed PADEM algorithm, and all four of these algorithms were implemented in $\mathrm{C}++$.

As a typical counting-based algorithm, SIENA can filter out the unmatched subscriptions as early as possible. Moreover, [23] also proposed a useful optimization strategy by using a selectivity table. Considering that the selectivity table is generally used in a pre-processing procedure before event matching, it can also be adopted in other counting-based algorithms such as TAMA and PADEM. In our experiments, the number of pre-processing rounds of the selectivity table was set to 5 to ensure fairness in the comparison.

TAMA is an approximate event matching algorithm based on a three-layer hierarchical index structure. The first layer of the index structure is a linear table indexed on the attributes, and the second layer of the index structure is also a linear table indexed on the discrimination levels. In addition, the third layer is a hash table indexed on the $I D$ of cells. Therefore, the event matching process is converted into a table look-up and checking process based on the counting-based algorithm. In our experiments, the discrimination level for TAMA was set to 13.

The index structure of REIN consists of multiple bucket lists. The bucket lists are indexed on attributes and constructed by partitioning the value domains of attributes into several buckets. In all experiments, the number of buckets for REIN was set to 1500 .

All parameters that may affect the performance of event matching algorithms are summarized in Table 5. To verify their influences on the matching performance, the values of these parameters in all experiments varied in different ranges. The value domain of each attribute was normalized on $[0,1.0]$. The values of constraints in subscriptions and 
events were generated with a precision of $10^{-6}$ [27]. In addition, the other two factors to consider in our experiments are the distribution of constraint values and the distribution of attributes in subscriptions [26].

\subsection{Matching Time}

The efficiency of event matching is the most important issue for event matching algorithms in content-based publish/subscribe systems, which can be expressed with the average matching time. Because many parameters can affect the efficiency of event matching, a series of experiments were conducted to reveal the impacts of such parameters. During each experiment, one thousand events were used as the input to measure the average matching time.

\subsubsection{Effects of the Number of Subscriptions}

The first experiment was designed to verify the effects of the number of subscriptions. According to the theoretical analysis in Section 4, the matching time would increase with the number of subscriptions $P$. The effects of the number of subscriptions on matching time are shown in Figure 5, where $N=75, M=15, C=10, w=0.5$, and the number of subscriptions participating in the experiments ranged from 200k to 2000k. When the width of range constraints $w$ was fixed, the lower bounds of range constraints were selected randomly from the range $[0,1-w]$, and the upper bounds were selected from the range $[w, 1.0]$. This means that the distribution of constraint values obeyed the uniform model. Similarly, the attributes contained in the subscriptions were randomly selected from the attribute space, which means that the distribution of attributes also obeyed the uniform model.

As shown in Figure 5, the matching time of all algorithms increases with the number of subscriptions, which confirms to the conclusion of the theoretical analysis. When the number of subscriptions increases, SIENA and TAMA are forced to examine more partially matched subscriptions than PADEM, which results in an increase in the matching time. By using the proposed index structure based on pairwise attribute subspaces, PADEM handles event matching more efficiently and avoids useless matching processes in the subspaces that are not related to the events. Therefore, PADEM outperforms SIENA and TAMA in terms of matching time under the same number of subscriptions. When the number of subscriptions is 2000k, PADEM is almost 10.1, 4.2, and 1.6 times faster than REIN, SIENA, and TAMA, respectively.

In addition, the matching time of REIN is longer than that of the other three algorithms under the same number of subscriptions, which is quite different from the experimental results in [27]. This phenomenon is caused by the different condition adopted in our experiments. In [27], all experiments were performed under the condition that the number of attributes in the attribute space is equal to that in events; therefore, REIN was more efficient than TAMA and SIENA. However, it is unlikely that an event contains all attributes of the entire system, particularly for largescale publish/subscribe systems. Therefore, our experiments were conducted under the condition that the number of attributes in the attribute space is considerably larger than that in events $(N \gg M)$. With this condition, REIN has to mark all subscriptions stored in the bucket lists that correspond to attributes that do not appear in the event, which means that a considerable amount of time is consumed. 


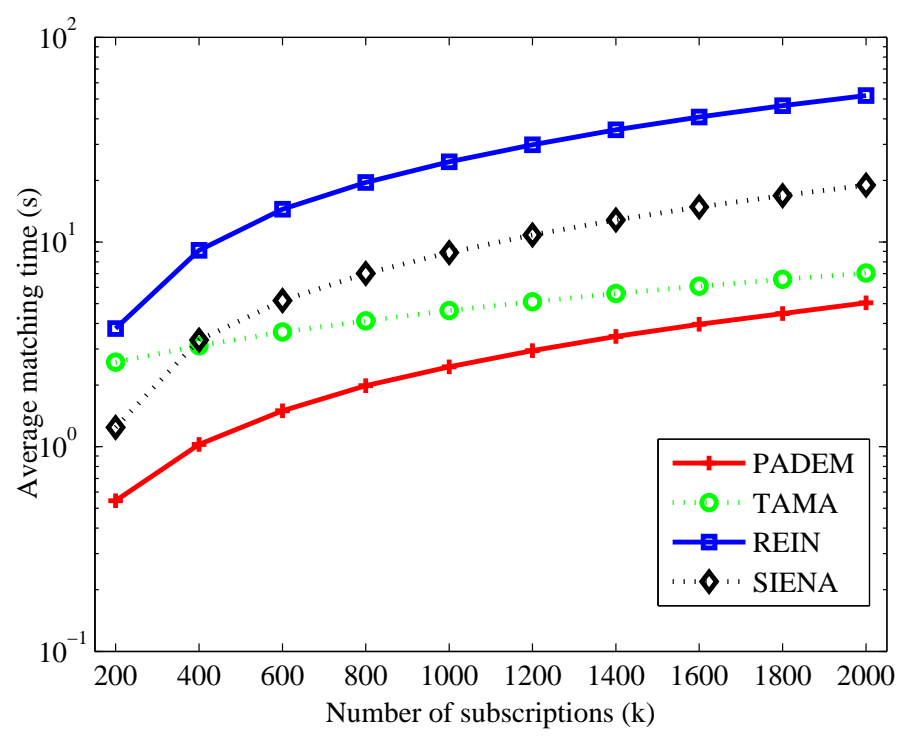

Figure 5: The average matching time versus the number of subscriptions.

\subsubsection{Effects of the Number of Attributes in Events}

The number of attributes in events, which is also called the size of the event space, is another parameter that may impact the matching time. An experiment was performed to verify the effects of the number of attributes in events. During this experiment, the parameters were set to $N=75, C=10, P=1000 \mathrm{k}$, and $w=0.5$; the number of attributes in events $M$ was greater than $C$ and ranged from 10 to 20; and the distribution of attributes in subscriptions and constraint values obeyed the uniform model. The experimental results are presented in Figure 6.

An increase in the number of attributes in events means that increasingly more constraints are satisfied with the event. As shown in Figure 6, the matching time of PADEM, SIENA and TAMA increases with the number of attributes in events. However, PADEM still achieves the best performance in terms of event matching under the same conditions. The reasons for this advantage can be explained by the characteristics of the novel index structure in PADEM, which can reduce the number of subscriptions involved in the event matching process. When the number of attributes in events is set to $M=20$, PADEM is 5.8, 3.2, and 1.6 times faster than REIN, SIENA, and TAMA, respectively, when matching one thousand events.

Remarkably, the matching time of REIN decreases as the number of attributes in events increases, which is quite different from the other three algorithms. The reason for this phenomenon is described as follows. When the number of attributes in events increases, the number of bucket lists that correspond to the attributes that do not appear in the events decreases. Therefore, the number of unmatched subscriptions that need to be marked in REIN also decreases, which also reduces its event matching time. These experimental results also conform to the conclusion of the theoretical analysis on matching time of REIN, which is described in Eq. (6). 


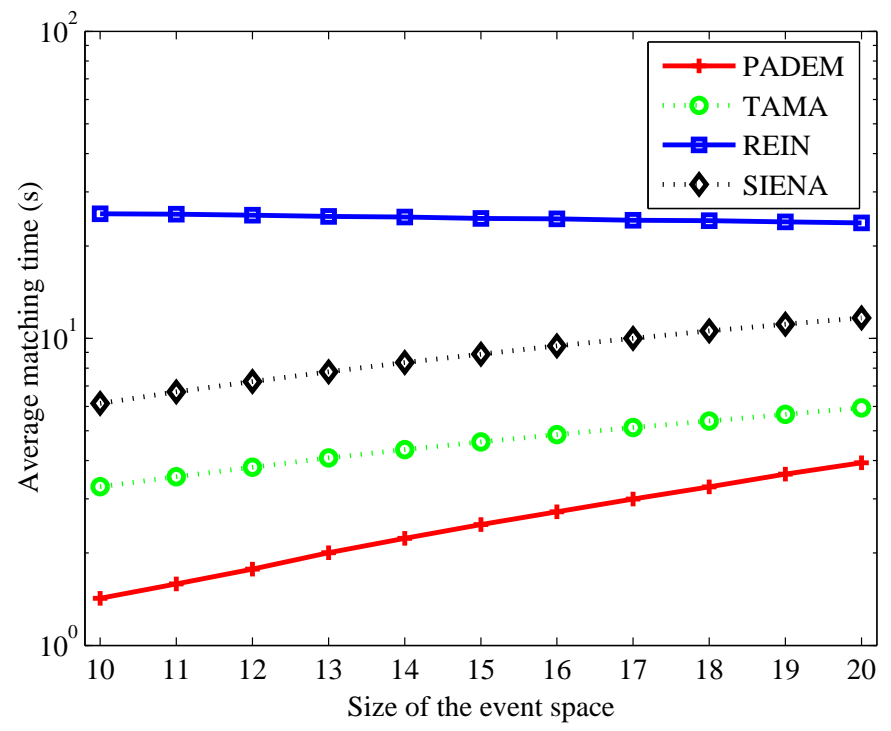

Figure 6: The average matching time versus the number of attributes in events.

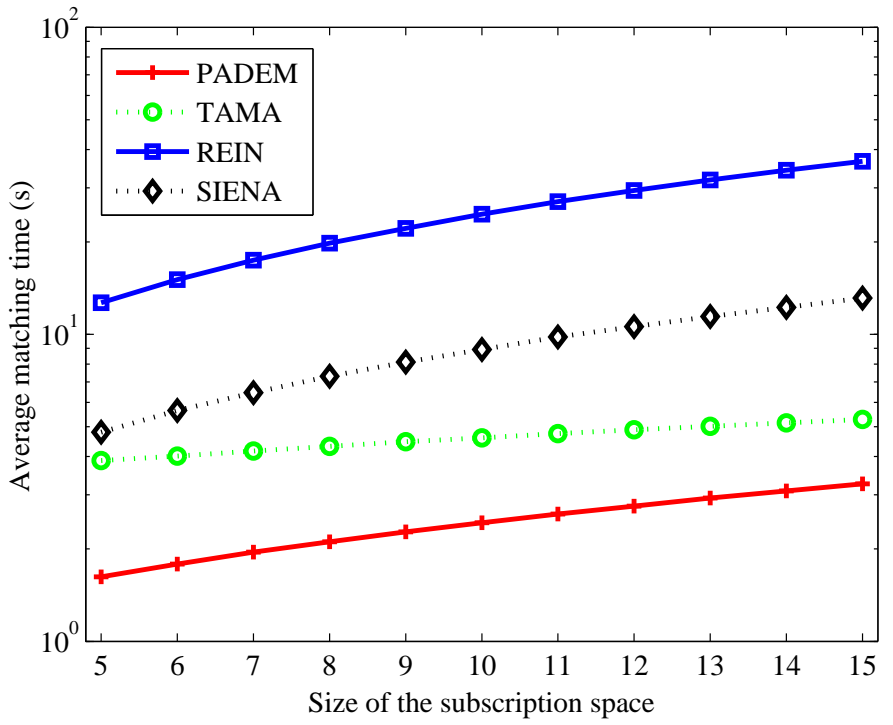

Figure 7: The average matching time versus the number of constraints in subscriptions. 


\subsubsection{Effects of the Number of Constraints in Subscriptions}

Another experiment was designed to observe the impact of the number of constraints in subscriptions (also called the size of the subscription space). In this experiment, the parameters were set to $N=75, M=15, P=1000 \mathrm{k}$, and $w=0.5$; the number of constraints in subscriptions $C$ ranged from 5 to 15 ; and the distribution of attributes in subscriptions and constraint values obeyed the uniform model. The results of this experiment are presented in Figure 7.

It is clear that the number of constraints satisfied or not satisfied with the events increases with the number of constraints in subscriptions, which means that SIENA, TAMA and PADEM have to count more matched constraints while REIN also has to mark more unmatched constraints. Therefore, the increase in the matching time in SIENA and TAMA results from the increase in partially matched subscriptions, which leads to an increase in the time complexity. PADEM achieves more efficient performance than the other three algorithms in terms of filtering out all unmatched subscriptions stored in irrelevant subspaces. When the size of the subscription space is 15, PADEM is 10.6, 4.2, and 1.7 times faster than REIN, SIENA, and TAMA, respectively.

\subsubsection{Effects of the Number of Attributes in the Attribute Space}

In large-scale content-based publish/subscribe systems, any attribute that appears in an event or a subscription belongs to the attribute space. Therefore, the number of attributes in the attribute space is another parameter that can affect the performance of event matching algorithms. The experiment for evaluating the effects of the number of attributes in the attribute space was conducted with the following parameters: $M=15, C=10, P=1000 \mathrm{k}$, and $w=0.5$; the number of attributes in the attribute space $N$ ranged from 50 to 100; and the distribution of attributes in subscriptions and constraint values obeyed the uniform model. The results of this experiment are presented in Figure 8.

As shown in Figure 8, the matching time of REIN is almost not influenced by the increase in the number of attributes in the attribute space. The reason for this result is due to the balance between the number of bucket lists and the number of subscriptions in each bucket list. Although the number of bucket lists increases with the number of attributes in the attribute space, the number of subscriptions in each bucket list also decreases. Therefore, the total time consumption on marking unmatched constraints in REIN maintains a high level.

In addition, the increase in the number of attributes in attribute space increases the number of buckets in SIENA, TAMA and PADEM, which also causes a decrease in the number of subscriptions stored in each bucket. Therefore, the matching time of these algorithms decreases as the attributes in attribute space increases. Among these algorithms, the matching time of PADEM decreases most sharply because its index structure contains more buckets. As shown in Figure 8, when $N=100$, PADEM is $13.4,4.2$ and 2.5 times faster than REIN, SIENA and TAMA, respectively. 


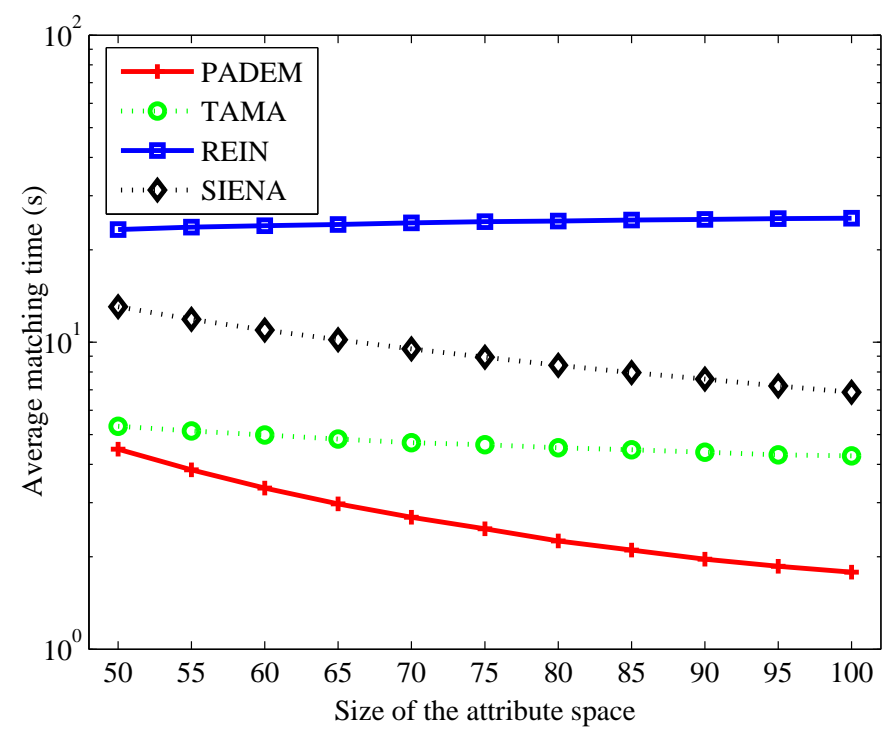

Figure 8: The average matching time versus the number of attributes in attribute space.

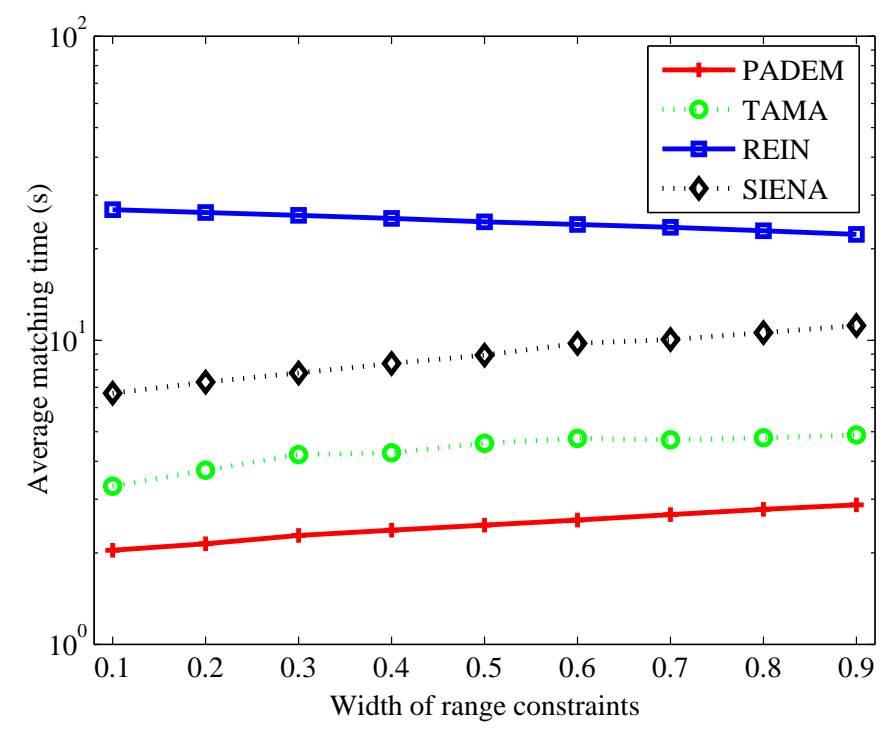

Figure 9: The average matching time versus the width of range constraints. 


\subsubsection{Effects of the Width of Range Constraints}

The width of range constraints determines the selectivity of subscriptions, which may affect the performance of event matching [27]. The experiment for evaluating the effects of the width of range constraints was designed with the following parameters: $N=75, M=15, C=10$, and $P=1000 \mathrm{k}$; the width of range constraints $w$ ranged from 0.1 to 0.9 ; and the distribution of attributes in subscriptions and constraint values obeyed the uniform model. The results of this experiment are presented in Figure 9.

As the width of range constraints increases, increasingly more constraints are satisfied with the events; therefore, REIN spends increasingly less time on marking unmatched constraints. As shown in Figure 9, the matching time of REIN decreases as the width of range constraints increases. In contrast, SIENA, TAMA and PADEM continue the growth trend because they have to count increasingly more matched constraints. Regardless, PADEM achieves the best performance in terms of matching time among the four algorithms. As shown in Figure 9, when $w=0.6$, PADEM is 7.4, 4.0 and 1.8 times faster than REIN, SIENA and TAMA, respectively.

\subsubsection{Effects of the Distribution of Constraint Values}

To verify the effects of the distribution of constraint values on the efficiency of event matching, the constraint values were selected based on three different models, namely, the uniform, normal, and Pareto models. In this experiment, the normal model was constructed by setting the mean and the variance to 0.5 and 0.01 , respectively. For the Pareto model, the mean and the scale were set to 0.5 and 2, respectively. In addition, the other parameters in this experiments were set to $N=75, M=15, C=10$, and $w=0.5$; the number of subscriptions participating in the experiment $P$ ranged from 200k to 2000k; and the results are presented in Table 7. As shown in Table 7, the matching time of PADEM under the normal and Pareto models are almost the same as that under the uniform model. Therefore, the proposed PADEM algorithm is adaptive to the distribution of constraint values.

\subsubsection{Effects of the Distribution of Attributes in Subscriptions}

In some applications, the attributes in subscriptions are not uniformly selected from the attribute space; thus, some attributes appear more frequently than others. A number of studies have proven that the relative frequency with which web pages are requested follows Zipf's law [30-32]. Zipf's law states that the relative probability of a request for the $i$-th most popular page is proportional to $1 / i$.

Therefore, another experiment was conducted for evaluating the effects of the distribution of attributes in subscriptions. The parameters in this experiment were set to $N=75, M=15, C=10$, and $w=0.5$; the number of subscriptions participating in the experiments $P$ ranged from 200k to 2000k; and the distribution of attributes in subscriptions was generated according to the Zipf distribution with the parameter $\alpha=1$. The results of this experiment are shown in Figure 10.

Compared with the results of the uniform distribution shown in Figure 5, the matching time of PADEM, SIENA and TAMA under the Zipf distribution increases to a certain extent. When the distribution of attributes in subscrip- 
Table 7: The average matching time versus the distribution of constraint values.

\begin{tabular}{cccc}
\hline Number of Subscriptions(k) & Uniform(s) & Normal(s) & Pareto(s) \\
\hline 200 & 0.55 & 0.56 & 0.53 \\
400 & 1.01 & 1.03 & 1.03 \\
600 & 1.50 & 1.49 & 1.52 \\
800 & 1.97 & 1.97 & 1.95 \\
1000 & 2.46 & 2.48 & 2.47 \\
1200 & 2.95 & 2.94 & 2.95 \\
1400 & 3.46 & 3.42 & 3.50 \\
1600 & 3.94 & 3.92 & 3.93 \\
1800 & 4.47 & 4.47 & 4.46 \\
2000 & 5.06 & 5.02 & 5.05 \\
\hline
\end{tabular}

tions obeys the Zipf model, some attributes appear more frequently in subscriptions, and this leads to an increase in the matching time cost on those attributes. In contrast, the increase in the number of some attributes in subscriptions also causes a reduction in the number of other attributes in subscriptions. Therefore, the number of unmatched subscriptions that need to be marked in REIN decreases. As shown in Figure 10, with the same number of subscriptions, the matching time of REIN under the Zipf distribution is less than that under the uniform distribution.

\subsection{Insertion Time}

For SIENA, REIN and PADEM, the information of a subscription (ID and constraint values) is stored $2 C$ times in their index structures, where $C$ is the number of constraints in this subscription. As mentioned above, the constraint values of the subscriptions in these algorithms are stored in ascending order. Therefore, the insertion time of these algorithms is proportional to the number of subscriptions that are already stored in their index structure. For TAMA, the times to store the $I D$ of a subscription depend on the width of range constraints $w$. When $w=0.5$, the $I D$ is stored $10 C$ times in its index structure at a minimum.

The experiment was conducted by inserting different numbers of subscriptions under the conditions that $N=75$, $M=15, C=10$, and $w=0.5$. Figure 11 shows the effects of the number of subscriptions on the insertion time. Clearly, PADEM achieves the best performance on inserting subscriptions, which confirms the positive effect of using pairwise attribute subspaces to store subscriptions. When the number of subscriptions is 2000k, the insertion time of SIENA, REIN and TAMA is 11.8, 2.5, and 6.2 times of PADEM, respectively. Therefore, the proposed PADEM algorithm is more efficient on satisfying the requirements of frequently updating. 


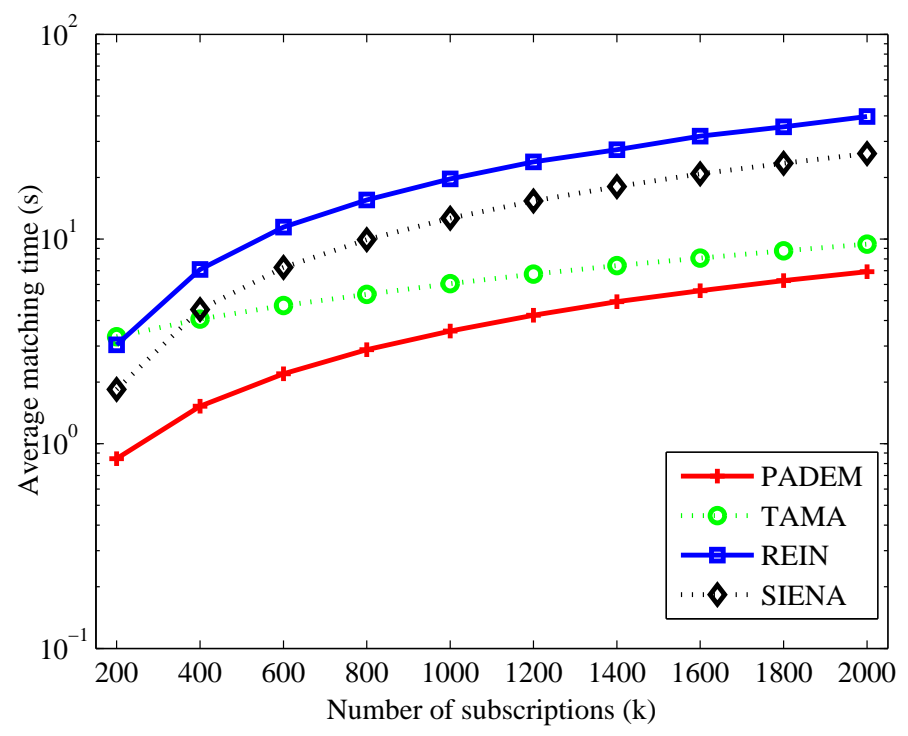

Figure 10: The average matching time versus the number of subscription (Zipf distribution).

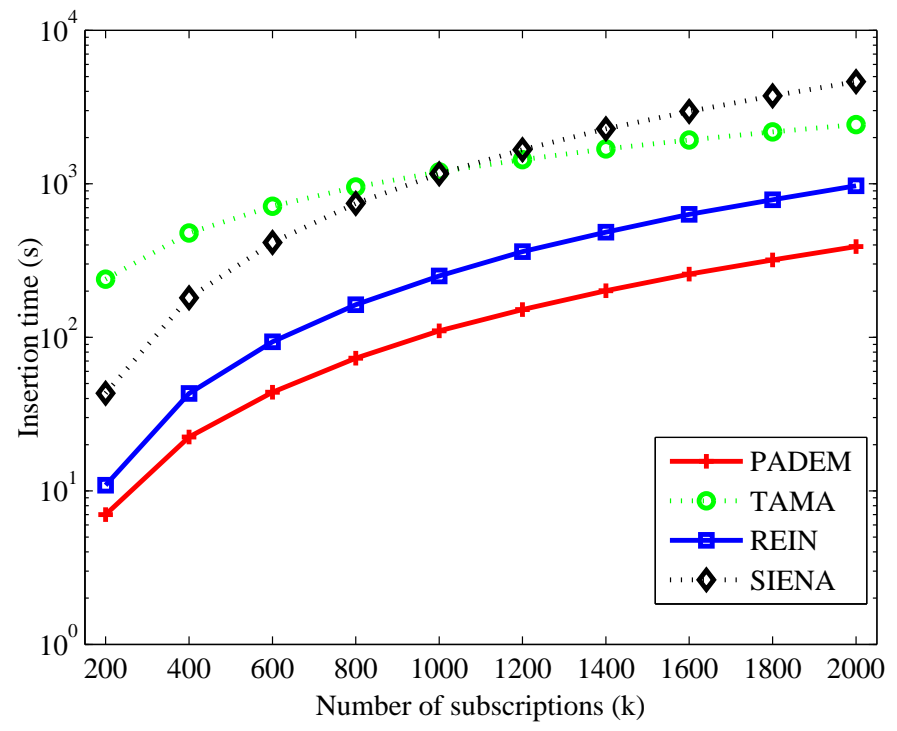

Figure 11: The insertion time versus the number of subscriptions. 


\subsection{Deletion Time}

Once the $I D$ of a subscription is given, the deletion process of a subscription is described as follows. First, the content of the subscription is selected from the subscription set. Then, the buckets that store this subscription are achieved. Finally, the information of the subscription is removed from the buckets according to the traversing searching method.

To evaluate the deletion time of those algorithms, another experiment was conducted by deleting different numbers of subscriptions under the conditions that $N=75, M=15, C=10$, and $w=0.5$. Deletion time is defined as the time spent on deleting subscriptions, and the results of the experiment are shown in Figure 12. Clearly, PADEM achieves the best performance in terms of deleting subscriptions. When the number of subscriptions is 2000k, PADEM is 13.1, 2.6 and 9.1 times faster than SIENA, REIN, and TAMA, respectively, on deleting the same number of subscriptions. As indicated by the insertion time and deletion time of these algorithms, the proposed PADEM algorithm is more applicable for dynamic environments where the subscriptions are updated frequently.

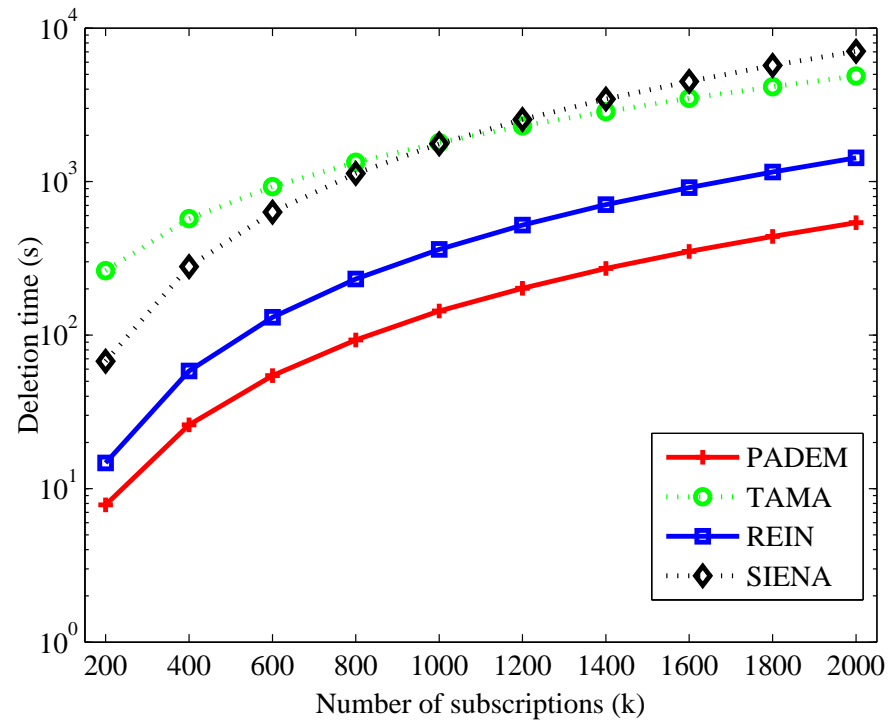

Figure 12: The deletion time versus the number of subscriptions.

\subsection{Memory Consumption}

Finally, the memory consumption for the above four algorithms is analysed as follows. Suppose that a constraint consists of a lower bound value and an upper value. Thus, the basic memory consumption of a constraint is 8 bytes. As analysed in Section 4.4, the subscription information is the most memory-consuming because the number of subscriptions $P$ is always larger than the number of attributes in the attribute space $N(P \gg N)$.

The results of the experiment on memory consumption are shown in Figure 13, where $N=75, M=15, w=0.5$, $C=10, d=11$, and $b=1500$. It is shown that TAMA consumes the most memory space because it has to store a 


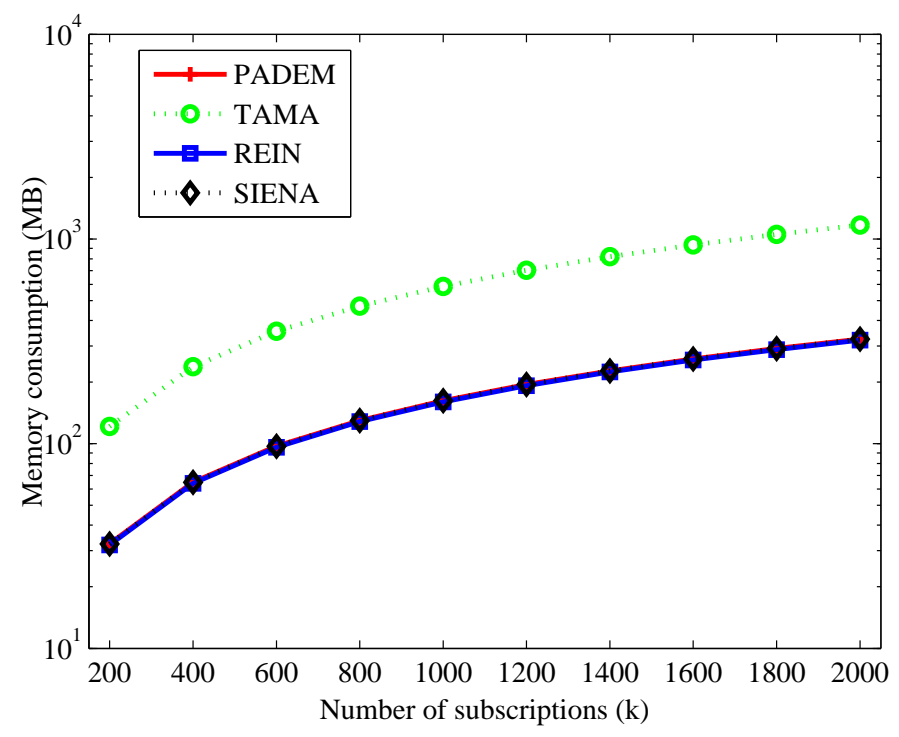

Figure 13: The memory consumption versus the number of subscriptions.

subscription more times than the other three algorithms. In contrast, REIN spends the least memory space because it does not need store the number of constraints in each subscription that is used for counting-based algorithms. PADEM consumes a little more space, approximately $1 \%$ more than REIN.

\section{CONCLUSION}

In terms of the efficiency of event matching in content-based publish/subscribe systems, an event matching algorithm named PADEM is presented in this paper. This algorithm constructs a structure based on multiple pairwise attribute subspaces and partitions all the subscriptions into attribute subspaces that have the same attributes. The method used to partition subscriptions is to ensure that the matching process in each subspace can only be triggered by events that contain the related attributes. When matching events, the algorithm only has to search the certain relevant attribute subspaces, which can avoid scanning of the entire subscription set. In this way, the number of subscriptions involved in the matching process is reduced and the efficiency of event matching is significantly improved. The experimental results show that PADEM has excellent performance in terms of matching time, insertion time, deletion time and memory consumption. Overall, PADEM is applicable for large-scale content-based publish/subscribe systems that contain high volumes of subscriptions along with numerous complex constraints, and it provides a highly potential solution for dynamic environments where the subscriptions are updated frequently. 


\section{Acknowledgements}

This work is supported by the Fundamental Research Funds for the Central Universities under Grant No.HIT.NSRIF. 2017014 and by the National Science Foundation of China under Grant No. 61501135. The authors would like to thank the editors and the reviewers for their helpful comments.

\section{References}

[1] T. E. Patrick, A. F. Pascal, G. Rachid, The many faces of publish/subscribe, ACM Computing Surveys 35 (2) (2003) $114-131$.

[2] X. Ma, Y. Wang, X. Pei, A scalable and reliable matching service for content-based publish/subscribe systems, IEEE Transactions on Cloud Computing 3 (1) (2015) 1-13.

[3] S. Ji, C. Ye, J. Wei, H.-A. Jacobsen, Towards scalable publish/subscribe systems, in: The 35th IEEE International Conference on Distributed Computing Systems (ICDCS'15)), IEEE, 2015, pp. 784-785.

[4] M. Yu, G. Li, T. Wang, J. Feng, Z. Gong, Efficient filtering algorithms for location-aware publish/subscribe, IEEE Transactions on Knowledge and Data Engineering 27 (4) (2015) 950-963.

[5] W. Rao, L. Chen, A. W.-C. Fu, H. Chen, F. Zou, On efficient content matching in distributed pub/sub systems, in: IEEE INFOCOM 2009, IEEE, 2009, pp. 756-764.

[6] K. Jayaram, W. Wang, P. Eugster, Subscription normalization for effective content-based messaging, IEEE Transactions on Parallel and Distributed Systems 26 (11) (2015) 3184-3193.

[7] Z. Shen, S. Tirthapura, S. Aluru, Indexing for subscription covering in publish-subscribe systems, in: ISCA PDCCS 2005, ISCA, 2005, pp. 328-333.

[8] X. Guo, J. Wei, D. Han, Efficient event matching in publish/subscribe: Based on routing destination and matching history, in: IEEE International Conference on Networking, Architecture, and Storage 2008 (NAS '08), IEEE, 2008, pp. 129-136.

[9] D. A. Tran, T. Nguyen, A random projection approach to subscription covering detection in publish/subscribe systems, in: IEEE International Conference on Collaborative Computing: Networking, Applications and Worksharing 2007, IEEE, 2007, pp. 362-369.

[10] G. Li, S. Hou, H. Jacobsen, A unified approach to routing,covering and merging in publish/subscribe systems based on modified binary decision diagrams, in: The 25th IEEE International Conference on Distributed Computing Systems (ICDCS'05), IEEE, 2005 , pp. $447-457$.

[11] Z. Liu, S. Parthasarthy, A. Ranganathan, H. Yang, Scalable event matching for overlapping subscriptions in pub/sub systems, in: The 2007 Inaugural International Conference on Distributed Event-based Systems, ACM, 2007, pp. 250-261.

[12] P. Triantafillou, A. Economides, Subscription summarization: A new paradigm for efficient publish/subscribe systems, in: The 24th IEEE International Conference on Distributed Computing Systems (ICDCS'04), IEEE, 2004, pp. 562-571.

[13] Y. Wang, L. Qiu, C. Verbowski, D. Achlioptas, G. Das, P. Larson, Summary-based routing for content-based event distribution networks, ACM SIGCOMM Computer Communication Review 34 (5) (2004) 59-74.

[14] H. Jafarpour, S. Mehrotra, N. Venkatasubramanian, M. Montanari, Mics: An efficient content space representation model for publish/subscribe systems, in: The 3rd ACM International Conference on Distributed Event-Based Systems, no. 7, ACM, 2009, pp. 1-12.

[15] X. Qin, J. Wei, W. Zhang, H. Zhong, A two-phase approach to subscription subsumption checking for content-based publish/subscribe systems, in: The 24th IEEE International Conference on Advanced Information Networking and Applications, IEEE, 2010 , pp. 1278-1285.

[16] A. M. Ouksel, O. Jurca, I. Podnar, K. Aberer, Efficient probabilistic subsumption checking for content-based publish/subscribe systems, in: The 7th ACM/IFIP/USENIX International Conference on Middleware, ACM, 2006, pp. 121-140.

[17] H. Jafarpour, B. Hore, S. Mehrotra, N. Venkatasubramanian, Subscription subsumption evaluation for content-based publish/subscribe systems, in: The 9th ACM/IFIP/USENIX International Conference on Middleware, ACM, 2008, pp. 62-81.

[18] K. R. Jayaram, P. Eugster, Split and subsume: Subscription normalization for effective content-based messaging, in: The 31th IEEE International Conference on Distributed Computing Systems (ICDCS'11), IEEE, 2011, pp. 824-835. 
[19] Z. Shen, S. Tirthapura, Approximate covering detection among content-based subscriptions using space filling curves, Journal of Parallel and Distributed Computing 72 (12) (2012) 1591-1602.

[20] M. Sadoghi, H.-A. Jacobsen, Be-tree: An index structure to efficiently match boolean expressions over high-dimensional discrete space, in: The 2011 ACM SIGMOD International Conference on Management of Data, ACM, 2011, pp. 637-648.

[21] D. Zhang, C. Chan, K. Tan, An efficient publish/subscribe index for e-commerce databases, VLDB Endowment 7 (8) (2015) $613-624$.

[22] T. Banerjee, S. Sahni, Pubsub: An efficient publish/subscribe system, IEEE Transactions on Computers 64 (6) (2015) 1119-1132.

[23] A. Carzaniga, A. L. Wolf, Forwarding in a content-based network, in: The 2003 ACM conference on Applications, Technologies, Architectures, and Protocols for Computer Communications, ACM, 2003, pp. 163-174.

[24] A. Carzaniga, D. S. Rosenblum, A. L. Wolf, Design and evaluation of a wide-area event notification service, ACM Transactions on Computer Systems 19 (3) (2001) 332-383.

[25] Y. Zhao, J. Wu, Towards approximate event processing in a large-scale content-based network, in: The 31th IEEE International Conference on Distributed Computing Systems (ICDCS'11), IEEE, 2011, pp. 790-799.

[26] S. Qian, J. Cao, Y. Zhu, M. Li, H-tree: An efficient index structure for event matching in publish/subscribe systems, IEEE Transactions on Parallel and Distributed Systems 26 (6) (2015) 1622-1632.

[27] S. Qian, J. Cao, Y. Zhu, M. Li, Rein: A fast event matching approach for content-based publish/subscribe systems, in: IEEE INFOCOM 2014, IEEE, 2014, pp. 2058-2066.

[28] V. Muthusamy, H.-A. Jacobsen, Infrastructure-free content-based publish/subscribe, IEEE/ACM Transactions on Networking 22 (5) (2014) $1516-1530$.

[29] T. You, K. Yang, C. Du, D. Zhong, Z. Yian, Hybird event matching algorithm for content-based publish/subscribe system, Acta Electronica Sinica 43 (2) (2015) 358-364.

[30] B. Lee, C. Pei, F. Li, P. Graham, S. Shenker, Web caching and zipf-like distributions: evidence and implications, in: IEEE INFOCOM 1999, IEEE, 1999, pp. 126-134.

[31] L. A. Adamic, B. A. Huberman, Zipfs law and the internet, Glottometrics 3 (1) (2002) 143-150.

[32] N. Maurizio, S. Claudia, Rank-size distribution of teletraffic and customers over a wide area network, European Transactions on Telecommunications 17 (4) (2006) 415-421. 